\title{
Exoskeleton to Rehabilitate Paralyzed Arm Based on Patient Healthy Arm Guidance
}

\author{
Jiajia Hu, Xinmin Xu, and Weidong Liu
}

\begin{abstract}
In this paper we discuss the stroke rehabilitation by robotic device and a novel exoskeleton, which is based on the patient's self-guided control will be presented. It is realized by moving the patient's own healthy arm in order to provide the movement trajectories for the exoskeleton. The main advantage of the exoskeleton is its flexibility and adaptivity for individual patient's rehabilitation training. A new model predictive control (MPC) algorithm for handling coupling and nonlinearity is also proposed and a microprocessor-based drive system for the exoskeleton will be adopted. In addition, to measure the angular positions corresponding to the joint angles of arm high resolution potentiometers are used.
\end{abstract}

Index Terms-Stroke rehabilitation, exoskeleton, model predictive control, motor control.

\section{INTRODUCTION}

Stroke is a medical emergency which can cause permanent neurological damage, complications, and lead to death. It is a rapid development leading to the loss of brain functions due to disturbance in the blood supply to the brain. As a result, the affected area of the brain is unable to function, which might result in an inability to move one or more limbs on one side of body. The World Health Organization (WHO) estimates that about 15 million individuals suffer from stroke every year around the world. At least one-third of them will require rehabilitation from disabilities after a stroke while another five million of these people will die. It is the third leading cause of death and the leading cause of serious long term disability in the world.

Stroke rehabilitation is the process by which patients with disabling strokes undergo treatment to help them return to normal life as much as possible. This process is usually supported by one rehabilitation team which is multidisciplinary as it involves staff with different skills working together to assist patients. Obviously, this traditional means of rehabilitation is extremely resource-limited and time-consuming, resulting in inadequate therapeutic practice received by patients.

In the field of Bio-Engineering, one of most important goals is to develop technology and systems for patients with motor impairment. Rehabilitation robotics research is dedicated to the understanding and augmenting rehabilitation

Manuscript received December 1, 2012; revised February 3, 2013.

Jiajia Hu and Xinmin Xu are with Department of Information Science and Electronic Engineering, Zhejiang University, 310027, China (e-mail: hujiajia131@gmail.com,xuxm@zju.edu.cn).

Weidong Liu is with Department of Electronic and Computer Engineering, Ngee Ann Polytechnic, 599489, Singapore (e-mail: lwe6@np.edu.sg). through the application of robotic devices. The integrated attributes of these devices, such as advanced control algorithms, human-robot interaction, and non-fatigue, etc., make it possible for patients to perform enough therapeutic training and some tasks in self-determined daily life with less help from others.

Over the past years development of robotic devices for rehabilitation has been received great attention and some products are available in the market. For example, in [1]-[6] they presented their new mechanical design and control schematics. However, most of these devices are commonly fixed on to a stable platform/structure (or it is stationary type) and provide simple and limited therapeutic training. Although many research and development works on robotic devices are on-going, they have not considered patient's self-guided control. For such devices, the functions provided will be limited and might have problem in adapting to individual patient's therapeutic training. To overcome the above disadvantages, in this paper a novel exoskeleton will be presented, which is for paralyzed arm rehabilitation exercise based on guidance from stroke patient's own healthy arm. It is noticed that most stroke patients actually lost temporally or permanently their limb movements on one half of the body; by contrast, the limb on the other half body is still healthy. In this paper we will focus on this group of stroke patients and one novel exoskeleton will be developed. The proposed exoskeleton system is designed to drive the paralyzed arm to move based on the movement trajectories and patterns generated by the healthy arm of patient. Therefore, it can be well adapted to individual patients as the intensity and range of the therapeutic practice are actually under control by patients. This feature leads to a safe, easy-use and patient-self-guided rehabilitation scheme. This paper is organized as follows: in section II, a new exoskeleton control method and a simple prototype for verification are presented. A new model predictive control algorithm for handling the nonlinear and coupling system is proposed in section III. In section IV, the implementation of the control system is mentioned and finally conclusions are given in section $\mathrm{V}$.

\section{EXoskeleton CONTROL METHOD}

\section{A. Structure of Exoskeleton}

The exoskeleton system is to be realized through the acquisition of joint angles, angular velocities and joint accelerations generated from patient's own healthy arm. With the aid of model-predictive control (MPC) algorithm and microprocessor-based drive system, the exoskeleton 
mounted on the paralyzed arm is controlled to perform movements. Fig. 1 illustrates this scenario, where the left arm generates the movement trajectory and pattern as it is supposed to be the healthy arm, while the exoskeleton mounted on the paralyzed right arm drives it to perform movements.

The exoskeleton assisting patients for the repetitive therapeutic rehabilitation and perform arm-movement in 3-D space. It is one of the expected products of new assistive technology in robotics, which is a powered mobile machine consisting primarily of an exoskeleton-line framework and a power supply that supplies at least part of the activation-energy for extremity movement. In order to reach the utmost efficiency and avoid injury to patients, the exoskeleton's kinematic chain should map on to patients limb anatomy, with multi-segment and joints corresponding to those of patients it is externally coupled with. Moreover, the exoskeleton will be designed to possess multi degrees-of-freedom to actuate the device to extend the arm and move it in 3-D space.

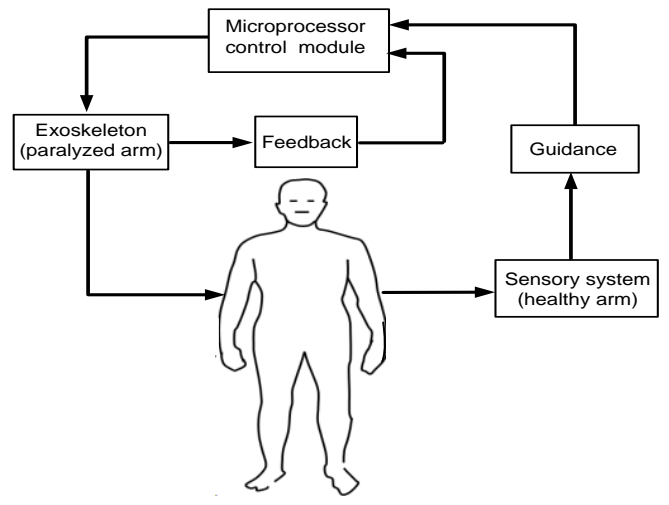

Fig. 1. Schematic diagram of arm exoskeleton with healthy arm guidance.

Fig. 2 shows the structure of the exoskeleton for the paralyzed patient. The actuators drive the exoskeleton, i.e. generate torques, while the sensors are used to measure the corresponding angles in order to realize the feedback control. The actuators are based on servo motors with specified torques. The torques required are computed based on the weight of exoskeleton and arm weight.

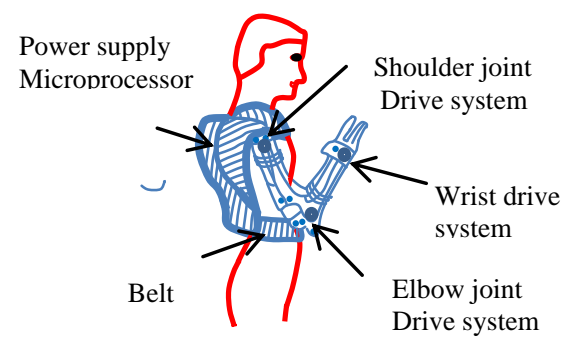

Fig. 2. The structure of the exoskeleton.

In the design of the system, all precautions and necessary specifications will be considered as much as possible. These include weight reduction of the exoskeleton to lower the mass moment of inertia, in order to ensure safety in operation, high reliability, smooth movement, comfort of wearing and easiness in maintenance, etc. Based on the knowledge of human upper-limb articulation, the exoskeleton mainly consists of a shoulder motion support part and an elbow motion support module. For each part, it mainly consists of arm link, driver, pulleys, DC motors (or pneumatic and/or hydro-actuators), force sensors, arm holders and the mechanism for moving centers of rotation of joints.

The novel exoskeleton for arm rehabilitation exercise based on the guidance from patient's own healthy arm mainly has two parts. The first part is responsible for guidance, or the generation of the movement trajectory, which is actually from the healthy arm of a patient, and can be obtained by sensory systems for the joint angles, generation of the movement trajectory. The second part is the artificial exoskeleton to drive the paralyzed arm to perform movements.

A simple two-segment exoskeleton prototype for verification has been built and shown in Fig. 3 .

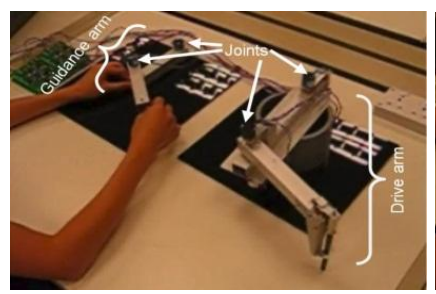

(a)

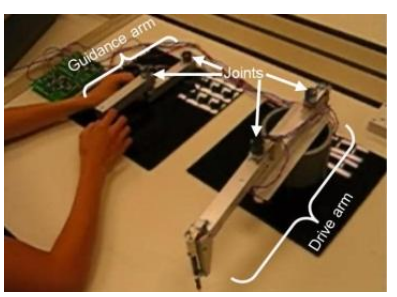

(b)
Fig. 3. Guidance arm and drive arm.

It consists of the guidance arm and the drive arm. Both have two joints and two segments representing upper arm and forearm. When the guidance arm moves around the joints, the drive arm accurately follows the same movement as the guidance arm.

Fig. 4 shows the test results for the prototype. They are the joint angles for both Shoulder and Elbow and are obtained by moving the shoulder part and elbow part of guidance arm. In this case the drive arm follows this movement in the same trajectory. It can be seen from the test result that the drive arm is able to follow the guidance arm by and large The errors of shoulder part and elbow part are quite small.

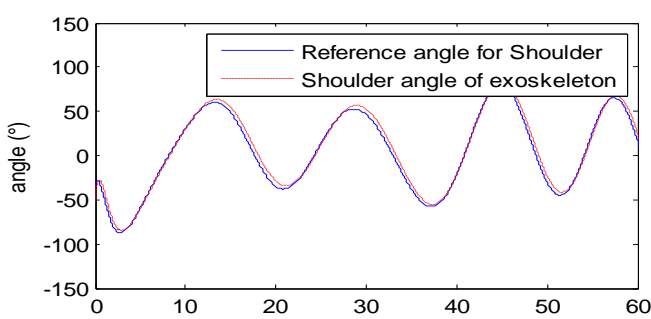

(a)

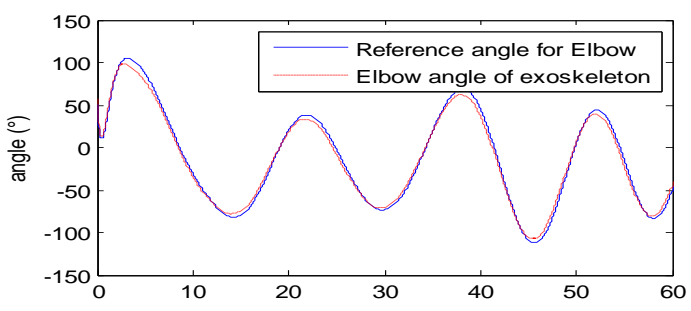

(b)

Fig. 4. The waveform of a simple two-segment exoskeleton.

\section{B. Sensor System}

The sensors are used to measure the corresponding angles in order to realize the feedback control and they are the same as those in the guidance. 


\section{1) Angle sensor circuits}

The joint angles are measured by using high resolution potentiometers. Note that:

- Shoulder has three potentiometers to measure rotations about the $\mathrm{x}$-axis, $\mathrm{y}$-axis and $\mathrm{z}$-axis.

- Elbow has two potentiometers to measure rotations about the $\mathrm{x}$-axis and $\mathrm{z}$-axis.

- Wrist has one potentiometer to measure the rotation about the $\mathrm{x}$-axis.

The potentiometers will be fixed on the surface of patients' guidance arm structure, being used to measure the changes in angles. When the angles of an arm change, it will cause a position change of sliding contact in the potentiometer, resulting in the change of potentiometer's voltage output. Then, the present voltage values (analog signals) are sent to Analog-to-Digital Converter (ADC), where these analog signals will be converted into digital signals. Finally, the processed signals will be sent to a microprocessor, where the controller is implemented. The potentiometer, ADC circuits and the microprocessor are as shown in Fig. 5.

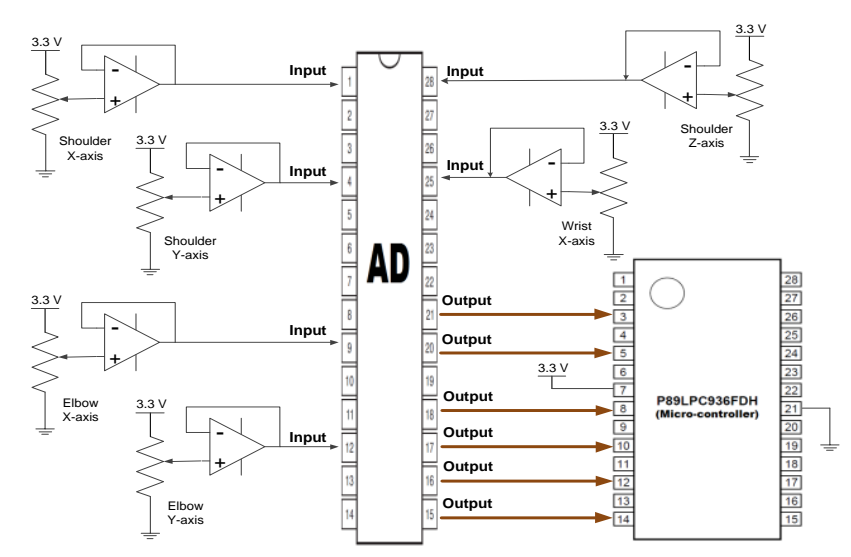

Fig. 5. Angle sensor circuits.

\section{2) Structure of sensory system}

The guidance arm wears a specially designed sensory system. It consists of a very thin carbon fiber link with a diameter of 10-15 mm. The joints of link can rotate:

- For shoulder, it rotates about the x-axis, y-axis and z-axis.

- For elbow, it rotates about the $\mathrm{x}$-axis and z-axis.

- For wrist, it rotates about the $\mathrm{x}$-axis.

The carbon fiber link is a semi-round or round shaped annulus, so the wires from sensors can be routed through the annulus holes to controller. To adapt to individual patients, the carbon fiber link length is adjustable through a ring screw.

The sensory system is attached along the healthy arm and a bandage is also used. And the sensory system has the advantage of light in weight and can be under the cloth sleeve. The bandage is used to tighten the link where the sensors are mounted on the healthy arm.

\section{CONTROL STRATEGY}

Owing to the fact that the exoskeleton and human body movements are very complex in nature, it is extremely important to deploy an optimal control strategy to manipulate the overall system dynamics during rehabilitation exercises. In this paper, Model Predictive Control (MPC) [7], [8] will be used for designing the feedback control system.

It is known that MPC is widely used and regarded as a robust control paradigm, referring to a class of computer control algorithm that utilizes an explicit process model to predict the future responses of the plant under control. The MPC algorithm considers plant behavior over a future horizon in time. This means that the effects of feedforward and feedback disturbances can be anticipated, thus allowing the controller to drive the plant more closely along a desired future trajectory. MPC is the dominant algorithm for centralized multivariable control, which is the optimal control strategy for complicated plants with multi-input and multi-output, such as human musculoskeletal movements. Considering the exoskeleton system is nonlinear and multi-input and multi-output system, we proposed a new MPC algorithm which might effectively handle its nonlinearity and coupling.

In the exoskeleton system, the data acquired from the sensory system mounted on the healthy arm will be sent to the controller as shown in Fig. 2. The controller performs the following aspects: Supervision; Control algorithm computation; Control decision making. The block diagram is shown in Fig. 6.

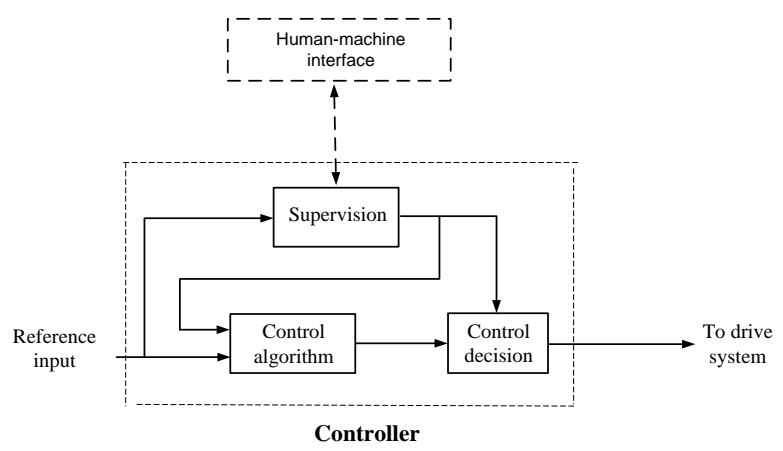

Fig. 6. The block diagram of controller.

\section{A. Supervision}

Roles of supervision:

- Validity check:

If the joint angles measured by the angle sensors are invalid, then reject the data and prohibit computation.

- Safety measurement:

Set the safe limits for joint angles and angular velocities to prevent injuries or make patient uncomfortable.

- Update the safety measurement:

\section{B. Control Decision}

Roles of control decision:

- Check if the control action from the control algorithm is reasonable, i.e., $\left|u_{i}(t)\right| \leq U_{i-\max }$, where $u(t)$ is the control action and $U_{i-\max }$ is the acceptable maximum control value. If it is unreasonable, reject take the control action and propos an action.

- The increment of the control action must be limited, i.e., $\left|\Delta u_{i}(t)\right|=\left|u_{i}(t)-u_{i}(t-1)\right| \leq \Delta U_{i-\max }$, where $\Delta u_{i}(t)$ is the increment and $\Delta U_{i-\max }$ is the acceptable maximum value. 


\section{Control Algorithm}

The control algorithm is to provide the control output which is then applied to the drive system so that the exoskeleton worn by patient will move and reach the target along the desired trajectory.

In this exoskeleton design, we have developed a control algorithm which is applicable to multi-input and multi-output systems. It is specially designed to compensate the effect of the gravity on the arm and exoskeleton, and also to achieve certain degree of decoupling. The detailed algorithm is as follows:

\section{1) Process and cost equation}

The dynamic model of the exoskeleton is a $n$-input and $n$-output nonlinear system and is described by the discrete-time model:

$$
y(t)=f_{p}\left[y(t-1), \ldots, y\left(t-n_{y}\right) ; u(t-1), \ldots, u\left(t-n_{u}\right)\right]+\xi(t)
$$

where $f_{p}: R^{\left(n \times\left(n_{y}+n_{u}\right)\right.} \rightarrow R^{n}$ is a nonlinear function. $y(t)$ and $u(t)$ are $n$-vectors of system output and input. $\{\xi(t)\}$ is a $n$-vector of white noise sequence.

We propose a modified dynamic system of (1) as follows:

$$
A\left(z^{-1}\right) y(t)=B\left(z^{-1}\right) u(t-1)+N(t-1)+\xi(t)
$$

where $A\left(z^{-1}\right)$ and $B\left(z^{-1}\right)$ are polynomial matrices in the backward shift operator $z^{-1}$ with $n_{A}<n_{y}, n_{B}<n_{u}$,

$$
\begin{aligned}
& A\left(z^{-1}\right)=I+A_{1} z^{-1}+\ldots+A_{n_{A}} z^{-n_{A}}, \\
& B\left(z^{-1}\right)=B_{0}+B_{1} z^{-1}+\ldots+B_{n_{B}} z^{-n_{B}} .
\end{aligned}
$$

$N(t)$ may denote some coupling elements and the load effect of the patient's arm and exoskeleton. Note that $N(t)$ can be identified mathematically on-line or off-line, for example, by using a neural network.

A quadratic cost function proposed for decoupling is as follows:

$$
\begin{aligned}
& \min J\left(N_{P}\right)=\min \left\{\sum_{j-1}^{N_{p}} \| \hat{y}(t+j)-r_{j} w(t+j)\right. \\
& \left.\quad+H_{j}\left(z^{-1}\right) N(t+j-1)\left\|_{I}^{2}+\sum_{j-1}^{N_{e}}\right\| u(t+j-1) \|_{\lambda_{i}}^{2}\right\} .
\end{aligned}
$$

Note that the cost function minimizes of the output predictions of the system. Here, $x_{Q}^{2}=x^{T} Q x . N_{p}$ and $N_{e}$ are the prediction and control horizons, respectively. $w(t)$ is a $n$-vector of reference signal and $r_{j}$ is the feedforward gain. $\lambda_{j}$ is the diagonal weighting factor matrix used to avoid an excessive control action. $H_{j}\left(z^{-1}\right)$ is a polynomial matrix to be determined, whose role is to remove the effect of $N(t)$ on the closed-loop system, which implies decoupling and/or compensation is achieved.

2) Output predictor

$$
\begin{aligned}
\hat{y}(t+j \mid t)= & G_{j}\left(z^{-1}\right) u(t+j-1)+E_{j}\left(z^{-1}\right) N(t+j-1) \\
& +F_{j}\left(z^{-1}\right) y(t)+L_{j}\left(z^{-1}\right) u(t-1)
\end{aligned}
$$

where $E_{j}\left(z^{-1}\right), F_{j}\left(z^{-1}\right), G_{j}\left(z^{-1}\right)$ and $L_{j}\left(z^{-1}\right)$ are the polynomial matrices determined by

$$
\begin{gathered}
I=E_{j}\left(z^{-1}\right) A\left(z^{-1}\right)+z^{-j} F_{j}\left(z^{-1}\right), \\
E_{j}\left(z^{-1}\right) B\left(z^{-1}\right)=G_{j}\left(z^{-1}\right)+z^{-j} L_{j}\left(z^{-1}\right), i=1,2, \ldots, N_{p} .
\end{gathered}
$$

\section{3) Control algorithm}

Solve (4) by applying (5) and after some manipulation the control algorithm is given by

$$
u(t)=\Lambda[R W-\tilde{F} y(t)-\tilde{L} u(t-1)-(\tilde{E}+\tilde{H}) N]
$$

where

$Y=\left[\begin{array}{c}\hat{y}(t+1 \mid t) \\ \hat{y}(t+2 \mid t) \\ \vdots \\ \hat{y}(t+j \mid t)\end{array}\right], U=\left[\begin{array}{c}\hat{u}(t) \\ \hat{u}(t+1) \\ \vdots \\ \hat{u}\left(t+N_{p}-1\right)\end{array}\right], N=\left[\begin{array}{c}N(t) \\ N(t+1) \\ \vdots \\ N\left(t+N_{p}-1\right)\end{array}\right]$

$$
\begin{gathered}
W=\left[\begin{array}{c}
w(t+1) \\
w(t+2) \\
\vdots \\
w\left(t+N_{p}\right)
\end{array}\right], \tilde{F}=\left[\begin{array}{c}
F_{1}\left(z^{-1}\right) \\
F_{2}\left(z^{-1}\right) \\
\vdots \\
F_{N_{P}}\left(z^{-1}\right)
\end{array}\right], \tilde{L}=\left[\begin{array}{c}
L_{1}\left(z^{-1}\right) \\
L_{2}\left(z^{-1}\right) \\
\vdots \\
L_{N_{P}}\left(z^{-1}\right)
\end{array}\right], \\
\tilde{E}=\operatorname{diag}\left[E_{j}\left(z^{-1}\right)\right], R=\operatorname{diag}\left(r_{j}\right), \\
\lambda=\operatorname{diag}\left(\lambda_{j}\right), \tilde{H}=\operatorname{diag}\left[H_{j}\left(z^{-1}\right)\right], \text { for } j=1,2, \ldots, N_{p} .
\end{gathered}
$$

\section{4) Comparison}

In the simulation, a PID controller is selected for a comparison.

Simulation results with the PID control and MPC are shown in Fig. 7. The control performance difference between the MPC and PID control can be observed. (a) and (c) show the output tracking unit step function. As a result, MPC achieved stability faster than PID control. Besides, when increasing the step function amplitude to 2, compared with PID, MPC could quickly achieve stability but also had no obvious overshoot as displaying in (b) and (d). Consequently, for the arm exoskeleton system model predictive control provides better control performance than the traditional PID 
control.

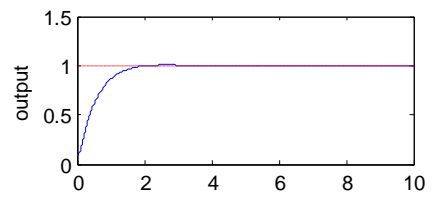

(a) PID

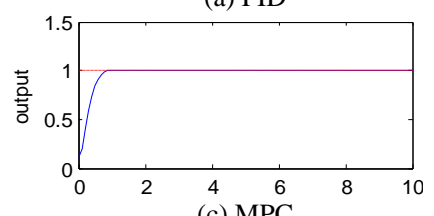

(c) MPC

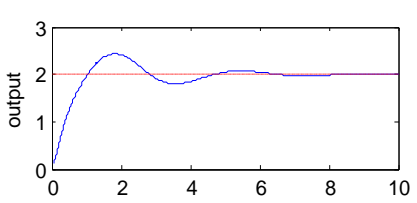

(b) PID

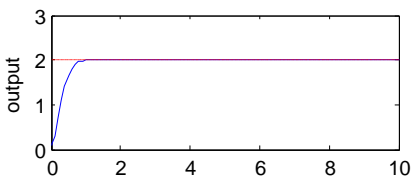

(d) MPC

Fig. 7. The simulation results of output.

\section{Electronics And Firmware Module}

The electronic circuit board will be built using reliable industrial components and is compatible with all relevant standards. The whole hardware part can be divided into several modules according to different functions, which includes Human-Machine-Interface, input/output devices, Micro-controller unit, power supply. The electronic circuits will be hybrid by design, containing analog and digital circuit elements. For the digital part, it is based on micro-controller unit (MCU) accompanied with firmware (low-level). Here the MCU will be mainly responsible for data acquisition, data analysis, data processing, data storage and so on.

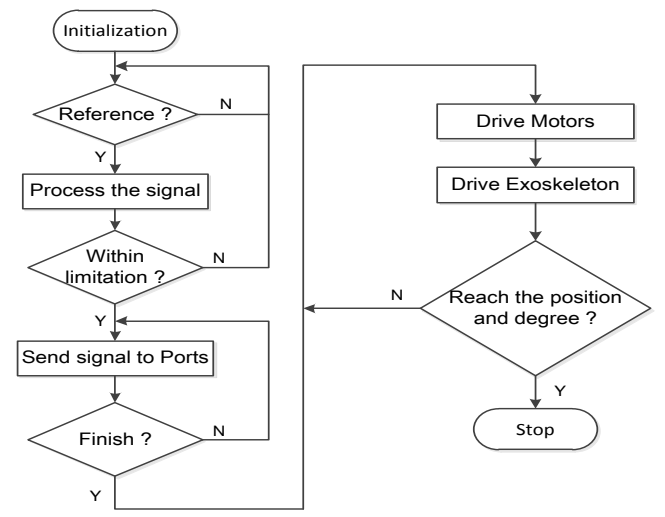

Fig. 8. The flowchart of firmware.

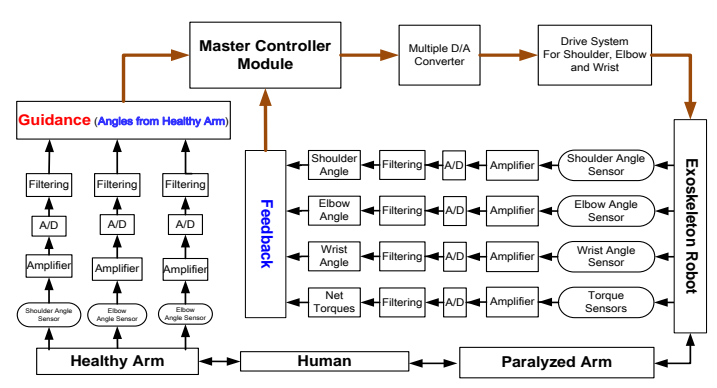

Fig. 9. The generalized block diagram of the exoskeleton.

The firmware framework is a hybrid multi-layer control architecture, which is tailored to the requirements of rehabilitation robot system. It is located in the memory of the Micro-Controller Unit and is mainly responsible for analyzing data from input devices, transmitting data/commands to output modules, supporting the operation

of human-machine-interface, fulfilling control strategies by command lines, etc. The firmware will be coded in $\mathrm{C}$ language, assembly language or both. The flowchart of firmware is shown in Fig. 8.

If additional complicated functions are required by the robot system, such as real-time supervision under medical staff, data storage and analysis of rehabilitation training by physiotherapists and occupational therapists, one additional computer system is needed. In this case, an additional high -level software and corresponding driver are to be added.

\section{CONCLUSION}

In this paper a novel exoskeleton is proposed. This exoskeleton provides for the stroke patients, who have lost the movement ability of one arm or too weak to do some movements, to perform the rehabilitation exercise based on guidance from patient's own healthy arm. In the help of the new model predictive control (MPC) system proposed in this paper, the exoskeleton can be controlled effectively. Fig. 9 shows the generalized block diagram of the microprocessor controlled exoskeleton. An initial prototype of the patient-guided exoskeleton has been built and tested. It can be seen from the results that the exoskeleton control schema presented here is more flexible and adaptive to individual patient's rehabilitation training. A more sophisticated version of prototype, which will be used for clinical tests is under building.

\section{REFERENCES}

[1] S. Parasuraman, A. W. Oyong, and V. Ganapathy, "Development of robot assisted stroke rehabilitation system of human upper limb," IEEE Annu. Conf. Auto. Scie. and Eng., pp. 256-261, Aug. 2009.

[2] P. R. Culmer, A. E. Jackson, S. Makower, R. Richardson, J. Alastair Cozens, M. C. Levesley, and B. B. Bhakta, "A Control Strategy for Upper Limb Robotic Rehabilitation With a Dual Robot System," IEEE/ASME Trans. Biomed. Eng., vol. 15, no.4, pp. 575-585, Aug. 2010.

[3] R. J. Sanchez, J. Y. Liu, S. Rao, P. Shah, R. Smith, T. Rahman, Steven C. Cramer, J. E. Bobrow, and D. J. Reinkensmeyer, "Automating Arm Movement Training Following Severe Stroke: Functional Exercises With Quantitative Feedback in a Gravity-Reduced Environment," IEEE Trans. Neural Sys. and Rehabil. Eng., vol. 14, no. 3, pp. 378-389, Sep. 2006.

[4] K. Kiguchi, R. Esaki, and T. Fukuda, "Development of A Wearable Exoskeleton for Daily Forearm Motion Assist," J. Advanced Robotics, vol. 19, no. 7, pp. 751-771, 2005.

[5] K. Kiguchi, M. H. Rahman, M. Sasaki, and K. Teramoto, "Development of a 3DOF mobile exoskeleton robot for human upper-limb motion assist," J. Robotics and Auto. Sys., pp. 678-691, 2008.

[6] Y. Mao and S. K. Agrawal, "Transition from Mechanical Arm to Human Arm with CAREX: a Cable Driven ARm EXoskeleton (CAREX) for Neural Rehabilitation," IEEE Annu. Conf. Robot. and Auto., pp. 2457-2462, 2012.

[7] D. W. Clarke, C. Mohtadi, and P. S. Tuffs, "Generalized predictive control-Part I. The basic algorithm," Automatica.

[8] D. W. Clarke, C. Mohtadi, and P. S. Tuffs, "Generalized predictive control-Part II Extensions and interpretations." Automatica.

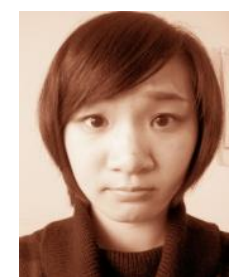

Jiajia $\mathrm{Hu}$ received Bachelor of Engineering degree in Dept. of Electronics and Information Engineering from Huazhong University of Science and Techology, China in 2011. She is currently a postgraduate in Information Science and Electronic Engineering Department of Zhejiang University, China. 


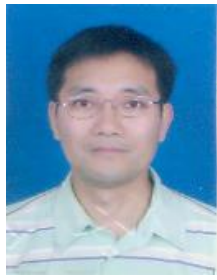

Xinmin Xu received the Doctor of Engineering degree in College of Electrical Engineering from Zhejiang University ,China in 2007. He is currently an associate professor in the Dept. of Information Science and Electronic Engineering, Zhejiang University, China. His main research interests include application of the embedded system, the microcomputer detection control, network integration, verification methodologies, FPGA dynamic reconfiguration theory, etc.. He has published academic papers nearly 50 in different levels, and taken charge dozens of research and development projects.

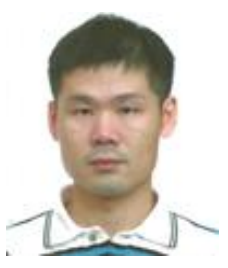

Weidong Liu received Bachelor of Engineering degree in Dept. of Control Technology from Harbin University of Science and Technology, China, in 2003; the Master of Science degree in School of Electrical \& Electronic Engineering from Nanyang Technological University, Singapore in 2007. He is currently a research engineer in Dept Electronic \& Computer Engineering, NGEE ANN POLYTECHNIC, Singapore. 